\title{
LA IMPORTANCIA DEL CLIMA EN EL MANEJO DE RESIDUOS DE COSECHA DEL ALGODONERO
}

\author{
Prause, Juan; Marinich, María Josefa
}

Facultad de Ciencias Agrarias. Universidad Nacional del Nordeste

Sargento Cabral 2131. (3.400) Corrientes, Argentina

\section{Resumen}

Las plagas de incidencia económica en el cultivo del algodonero, cumplen su ciclo normalmente, pero la invernación la realizan en el suelo o en los rebrotes de plantas de algodón, las que ofrecen condiciones de sobreviviencia y reproducción para que un mayor número de insectos puedan, en campañas agrícolas sucesivas, provocar daños futuros al cultivo. Las principales plagas del algodonero son la Lagarta Rosada (Pectinophora gossypiella); Broca del algodonero (Eutinobothrus brasiliensis) y Picudo del algodonero (Anthonomus grandis), todas ellas necesitan atravesar el período invernal en plantas hospederas específicas para cada insecto, en los residuos de la cosecha anterior y/o en el suelo. Su sobrevivencia depende directamente de los elementos del clima, principalmente precipitaciones, temperaturas y heladas.

Los objetivos del programa fueron: i) capacitar in situ a productores algodoneros en el manejo de los residuos de cosecha del algodonero, integrando los conceptos de cómo los elementos del clima juegan un importante papel en el manejo del suelo y la sanidad vegetal dentro del sistema productivo a pequeños y medianos productores; ii) difundir prácticas conservacionistas sencillas destacando los beneficios que se obtienen del manejo adecuado de los residuos de cosecha; iii) vincular la Universidad con el productor algodonero, alumnos de escuelas agropecuarias, y profesionales de la actividad pública y privada.

Las jornadas de tipo teórico-prácticas de 8 horas de duración no continuas, se realizaron en lotes de los productores algodoneros y se reparte en tres módulos que se constituyen en la base del programa de capacitación: clima: la importancia en la fenología de la planta, el manejo del suelo, la sanidad y la semilla. El impacto del proyecto reside en que se puede transferir a no sólo a productores algodoneros e incorporar a la currícula de las escuelas técnicas dentro de un módulo de extensión rural.

Palabras clave: clima, suelo, sanidad, semillas, rastrojos del algodonero.

\section{Antecedentes}

Se conoce que el hombre de campo trata de explicar su existencia mediante un aprendizaje basado en su propia experiencia. Esto le ha dado una gran diversidad de formas de saber y conocer que se van transformando en la medida en que se tienen nuevas vivencias, las cuales se van cristalizando, algunas veces, en teorías o principios y otras se guardan o transmiten en forma imperceptible de una generación a otra. Este aprendizaje se da en interacciones colectivas de comunicación, en la interacción entre padres e hijos, entre familias y vecinos, entre el hombre y su entorno. El fruto de este saber es producto de la convivencia hombre-naturaleza, hombre-medio. Sobre esta forma de aprender no se disponen de estudios que permitan comprender desde lo metodológico, cómo se desarrolla este conocimiento.

Las plagas de incidencia económica en el cultivo del algodonero, cumplen su ciclo normalmente, pero la invernación la realizan en el suelo o en los rebrotes de plantas de algodón, las que ofrecen condiciones de sobreviviencia y reproducción para que un mayor número de insectos puedan, en campañas agrícolas sucesivas, provocar daños futuros al cultivo. Las principales plagas del algodonero son la Lagarta Rosada (Pectinophora gossypiella); Broca del algodonero (Eutinobothrus brasiliensis) y Picudo del algodonero (Anthonomus grandis), todas ellas necesitan atravesar el período invernal en plantas hospederas específicas para cada insecto, en los residuos de la cosecha anterior y/o en el suelo.

Desde hace más de 50 años se estudia el problema de las chacras algodoneras, haciéndose hincapié en el estancamiento de la producción y el agotamiento y erosión de los suelos, como consecuencia del monocultivo, de la quema de los restos de cosecha y del exceso de labores culturales. Las prácticas conservacionistas implican el manejo de residuos de cosecha y el aporte de éstos residuos al suelo, constituyen un factor importante en el mantenimiento de las principales propiedades físicas y químicas del mismo, y la forma en que se realiza tal adición, tendrá efectos sobre la dinámica de la materia orgánica, del ciclo de nutrientes y del almacenamiento del agua en el suelo. Existen instrumentos legales que obligan a la destrucción anual de rastrojos, pero es

Publicado en formato digital: Dr. Juan Prause e Ing. Agr. María Josefa Marinich. LA IMPORTANCIA DEL CLIMA EN EL MANEJO DE RESIDUOS DE COSECHA DEL ALGODONERO. Revista Geográfica Digital. IGUNNE. Facultad de Humanidades. UNNE. Año 9. № 18. Julio - Diciembre 2012. ISSN 16685180 Resistencia, Chaco. En: http://hum.unne.edu.ar/revistas/geoweb/default.htm 
deficiente la capacitación para explicar los beneficios que acarrea una destrucción correcta en tiempo y forma, a pesar que está en vigencia el Decreto Nacional No 2017/57 el cual establece la obligación anual del desmenuzado de los rastrojos del algodonero.

El panorama actual de nuestro país, con miles de hectáreas de suelos que se degradan física y químicamente, muy erosionados y en algunos casos en situación irreversible por lo menos económicamente, nos exige que pensemos en soluciones a largo plazo. Esencialmente el manejo de los rastrojos, se lo recomienda como un eslabón más en la cadena del Manejo Integrado de Plagas (MIP), debiendo realizarse a través de la utilización de adecuadas prácticas agronómicas en tiempo y forma, en función de la calidad de la semilla, del manejo del suelo, y de la sanidad de la planta, considerando como eje integrador a la fenología de la planta, conformando en su conjunto el Manejo Integrado del Cultivo (MIC), dentro de un paquete de las buenas prácticas agrícolas.

Básicamente, se apunta a comenzar una campaña agrícola con el menor número de plagas en el lote; la incidencia económica que esta práctica trae aparejada, no se puede medir con exactitud, pero con solo evitar una aplicación de agroquímicos el beneficio ambiental y económico, está asegurado. Por otra parte, debemos tener en cuenta que la degradación del suelo causa graves problemas de rentabilidad en la producción agrícola, dado que la pérdida de nutrientes afecta principalmente la eficiencia de uso del agua y de nutrientes. Ante la necesidad de aumentar la eficiencia productiva surgen algunos cuestionamientos al sistema tradicional y aparece el manejo de la materia orgánica del suelo, en el centro de cualquier alternativa promisoria. Y desde el punto de vista social, la pérdida de la fertilidad física y química de los suelos, como consecuencia de la quema de la materia orgánica edáfica y del monocultivo algodonero, trae aparejado el empobrecimiento del productor y finalmente el abandono de las tierras que se vuelven improductivas, produciendo un problema social cada vez más notorio.

A pesar que la base económica de la región NEA es, y seguirá siendo todavía en un futuro previsible, el desarrollo rural y por consiguiente, la única oportunidad de progreso económico para los pequeños productores, será la capacitación agropecuaria no sólo de ellos sino también de sus hijos. Existe una proporción creciente de hijos de pequeños productores que alcanzando un nivel de escolaridad medio, salen de las escuelas rurales (no agrotécnicas), con esperanzas irrealizables de empleo urbano y con conocimientos para los que no hay demanda.

Definitivamente, las razones para adoptar la práctica del picado de los residuos de cosecha de los cultivos se encuadra dentro de un sistema productivo conservacionista, que está influido por razones ecológicas, económicas y sociales, y en consecuencia, se presenta como una alternativa válida para los productores agrícolas.

\section{Los objetivos del programa fueron:}

a.- Capacitar in situ a productores algodoneros en el manejo de los residuos de cosecha del algodonero integrando los conceptos de calidad de suelo, sanidad vegetal y genética de semillas, dentro del sistema productivo a pequeños y medianos productores.

b.- Difundir a nivel de predio rural, prácticas conservacionistas sencillas destacando los beneficios que se obtienen del manejo adecuado de los rastrojos, conformando en su conjunto un manejo integrado del cultivo.

c.- Vincular la Universidad con el productor algodonero, alumnos de escuelas agropecuarias, y profesionales de la actividad pública y privada.

Este proyecto fue desarrollado con todo éxito desde el año 1998 y hasta la fecha, dictándose más de 60 cursos de capacitaciones a pequeños y medianos productores algodoneros:

*.- En el marco del Programa La Universidad en el Medio de la Universidad Nacional del Nordeste (UNNE), avalado por productores beneficiados.

*.- Solicitado por las autoridades del Ministerio de La Producción de la Provincia del Chaco.

Publicado en formato digital: Dr. Juan Prause e Ing. Agr. María Josefa Marinich. LA IMPORTANCIA DEL CLIMA EN EL MANEJO DE RESIDUOS DE COSECHA DEL ALGODONERO. Revista Geográfica Digital. IGUNNE. Facultad de Humanidades. UNNE. Año 9. № 18. Julio - Diciembre 2012. ISSN 16685180 Resistencia, Chaco. En: http://hum.unne.edu.ar/revistas/geoweb/default.htm 
*-- Por Convenio SENASA-UNNE en el marco del Proyecto de Prevención y Erradicación de Focos de Picudo del Algodonero (Préstamo BID No 899/OC/AR) para el dictado de 10 capacitaciones en las Provincias de Corrientes, Chaco y Formosa durante los años 2004/2005.

*.- Solicitado por el Instituto de Investigación y Desarrollo Tecnológico para la Pequeña Agricultura Familiar del NEA (IPAF-NEA), dependiente del Instituto Nacional de Tecnología Agropecuaria (INTA), de Laguna NAINECK, Provincia de Formosa en los años 2007/2008.

*.- Capacitaciones a profesionales y productores del Proyecto Tapenagá de la Provincia del Chaco.

Más de 700 productores asistidos desde la iniciación de la capacitación, en el año 1996 a la fecha, en las provincias de Corrientes, Chaco y Formosa.

\section{Ha merecido las siguientes menciones:}

a.- Distinción a los investigadores participantes del trabajo sobre "Manejo de Residuos de Cosecha del Algodonero y su impacto en la Sanidad y los Suelos", por el impacto que su aportación científica ha causado en el IV Congreso Iberoamericano de Química y Física Ambiental, organizado por la Sociedad Iberoamericana de Física y Química Ambiental. Cáceres (Extremadura, España). Fecha: 22 al 26 de mayo de 2006.

b.- Premio "El Gauchito" de Plata y Diploma de Honor en el Rubro Facultades Relacionadas con el Sector Agropecuario, por el importante aporte al mantenimiento de los valores éticos, morales y de la cultura del trabajo, desde el sector agropecuario. Institución Otorgante: Fundación "El Gauchito".

Trabajo presentado: "Capacitación en Manejo de Residuos de Cosecha del Algodonero integrando Sanidad y los Suelos". Sede: Bolsa de Comercio de Rosario, Santa Fe. Argentina. Jurado: SAGPyA, SRA, INTA, AAPRESI, Coninagro, FAA, AACREA, Gobiernos de las provincias de Santa Fe, Córdoba y Buenos Aires. Rosario, Argentina. Fecha: 08 de diciembre de 2006.

\section{Metodología de trabajo.}

Para el pequeño productor algodonero no existe un lugar específico para el aprendizaje ni un cúmulo de conocimientos teóricos a los que se puede recurrir de una forma sistemática para encontrar cierta clase de información. Con frecuencia en los trabajos de capacitación, se confunde porque se cree que con sólo dar datos, los productores después podrán pensar y hacer deducciones para actuar. El peligro es que el productor se acostumbre a almacenar datos y actuar por imitación, y espere que quién le enseña determine si su proceder es correcto o incorrecto.

Mientras el productor, desde su propia perspectiva, no tenga suficientes razones para aceptar la información y decidir cómo actuar, el aprendizaje que llega será sólo mecánico, no modificará sus esquemas, será no racional y sin probabilidades de cambiar.

\section{Estrategias de trabajo}

Las jornadas de tipo teórico-prácticas de 8 horas de duración no continuas, se realizaron únicamente a campo, en lotes de los productores algodoneros de cada zona de influencia seleccionada. La duración de la jornada se reparte en tres módulos que se constituyen en la base del programa de capacitación: clima: la importancia en la fenología de la planta, el manejo del suelo, la sanidad y la semilla. El impacto del proyecto reside en que se puede transferir a no sólo a productores algodoneros e incorporar a la currícula de las escuelas técnicas dentro de un módulo de extensión rural.

a.- Suelos: se muestra la relación que existe entre el suelo y la planta a través de las raíces, que son extraídas del mismo lote en donde se desarrolla la capacitación. Se destaca la importancia de la forma de las raíces, extensión y deformaciones detectadas en las mismas, como una manera de introducir el problema de la fertilidad física a través de la pérdida de la estructura del suelo por disminución de la estabilidad de los agregados y la formación de pisos de arados y costras. En todo momento se hace hincapié en la importancia de la materia orgánica del suelo y de la cobertura vegetal en el control de la erosión del suelo, la formación de costras, y la pérdida de la fertilidad química del suelo.

Publicado en formato digital: Dr. Juan Prause e Ing. Agr. María Josefa Marinich. LA IMPORTANCIA DEL CLIMA EN EL MANEJO DE RESIDUOS DE COSECHA DEL ALGODONERO. Revista Geográfica Digital. IGUNNE. Facultad de Humanidades. UNNE. Año 9. № 18. Julio - Diciembre 2012. ISSN 16685180 Resistencia, Chaco. En: http://hum.unne.edu.ar/revistas/geoweb/default.htm 
La demostración práctica se realiza seleccionando diferentes agregados del suelo agotado por el monocultivo del algodonero y otros terrones extraídos de lugares próximos, debajo de alambrados, montes o potreros con muchos años de pasturas, en los cuales la vegetación protegió al suelo y se puede observar a través del cambio de color del mismo, la presencia de materia orgánica edáfica.

Se destaca el color más oscuro del suelo, con una mayor porosidad, huecos y canales debido a la presencia de raíces y fauna edáfica, que no se encuentra en el suelo proveniente del monocultivo.

Posteriormente se muestra el efecto de la acción de las gotas de agua sobre ambos terrones y cómo en el suelo de monocultivo la destrucción del terrón es más o menos rápida, el agua queda totalmente turbia como consecuencia del material que arrastra, destacándose que ese suelo es transportado a los sitios más bajos, pudiendo producir la inundación de una parte del lote.

Para reforzar la importancia de la materia orgánica edáfica se procede a sumergir en agua terrones de ambos suelos, mostrando cómo en el caso del agregado proveniente del monocultivo la destrucción del mismo es total, destacando que tanto por el impacto de la gota de agua de lluvia como, por la inundación del suelo, se produce la destrucción de los terrones, formando una capa dura en su superficie, cuando se seca.

Para lograr que el productor comprenda sobre la función de la materia orgánica en el suelo y porqué lo mantiene intacto, se le recuerda que para fabricar ladrillos y paredes de barro es necesario usar algún material orgánico como paja picada, cáscara de algodón, arroz, o aserrín, que hacen de "liga".

b.- Sanidad: Se enseña al productor sobre la búsqueda de plagas que invernan en los rastrojos, reconociendo el ciclo biológico de cada una de ellas y destacando la importancia de un buen picado del mismo para evitar la permanencia de las principales plagas del algodonero. En cada caso se les hace buscar y se muestran los diferentes órganos de la planta, en donde cada plaga produce el daño y pasa su período invernal. Se pone de especial énfasis en la demostración del uso de trampas con feromonas y el reconocimiento de otras plagas que se encuentren en el cultivo como así también, en la importancia de no abusar en la aplicación de agroquímicos para eliminarlas, porque también se eliminan los insectos predatores rompiendo el equilibrio biológico que debe existir en el cultivo.

Se muestran preparados entomológicos con los distintos estadios de crecimiento de cada plaga para que el productor las pueda reconocer posteriormente, a campo. En ambos casos se destaca la importancia del clima, especialmente temperatura y humedad, como elementos propicios para el desarrollo de plagas, y/o para acelerar la descomposición de los rastrojos del algodón a los fines de aumentar la materia orgánica edáfica.

c.- Semillas: Se destaca la importancia de la genética y calidad de la semilla para la siembra, puesto que los pequeños y medianos productores tienen la costumbre de guardar semilla de algodón de un año a otro y en muchos casos por varios años. De ahí parte la premisa de insistir en la conveniencia de emplear una buena semilla a los fines de partir con un buen stand de plantas en el cultivo, con un buen potencial de rendimiento. Se insiste en el tema de la calidad de la semilla, mostrándose diferentes clases de semillas de acuerdo a su conservación y se destaca la importancia de su origen y genética, y la importancia de conservar los rótulos que deben estar incorporados a las bolsas cuando se procede a la compra de las mismas, a los fines de asegurar la pureza varietal, y poder en caso de necesidad, reclamar ante los organismos competentes sobre las irregularidades que se pudieran haber presentado.

e.- Legal: Se insiste en informar sobre la vigencia del Decreto Nacional $N^{\circ} 2017 / 57$ con sus disposiciones anuales de desmenuzado de los rastrojos del algodonero, que obliga al productor a no dejar residuos de cosecha del algodonero en pié, a los fines del control de las plagas, comenzando la próxima siembra con el menor número de plagas en los lotes.

f.- Herramientas: para finalizar la jornada, se realizan demostraciones prácticas con diferentes herramientas, que operan en el terreno, para evaluar los distintos métodos de desmenuzados y la semi-incorporación de los rastrojos, de acuerdo a la zona y al nivel de productores (pequeños, medianos y grandes). El concepto común a los productores es que con la rastra de discos se pica

Publicado en formato digital: Dr. Juan Prause e Ing. Agr. María Josefa Marinich. LA IMPORTANCIA 
el rastrojo, y a los efectos de demostrar que esto no sucede, se procede a emplear este implemento agrícola sobre un residuo de cosecha y se invita a los productores a revisar los rastrojos luego de pasar la rastra de discos. Es la oportunidad de hacerles notar que cuando se pasa la rastra de discos aunque sea dos veces, siempre deja las plantas de algodón sin picarlas, están intactas y numerosos casos las plantas quedan fijadas al suelo (pudiendo rebrotar y florecer en el invierno) y en otros casos, hay una semiincorporación de la planta entera. Como consecuencia de ello, el suelo produce un abrigo orgánico que protege a las plagas que ya están en los tallos y cápsulas, facilitando la hibernación de las mismas.

\section{Impacto}

a.- Es un proyecto que se puede transferir no sólo a los productores algodoneros, sino también a cualquier otro productor agropecuario y es posible incorporar a la currícula de las escuelas técnicas y a las facultades de ciencias agropecuarias dentro como una cátedra optativa de extensión rural, debido que durante la capacitación se desarrollan tres temas fundamentales en agricultura. Los hemos denominado: "La importancia de prestar atención de las Tres S", refiriéndonos específicamente al cuidado del Suelo, Semilla y Sanidad.

Estos conceptos pueden y deben ser aplicados a cualquier cultivo que se trate, y como consecuencia de ello, se considera un programa transferible a las demás actividades agrícolas, tanto intensivas como extensivas.

b.- Dentro del marco de las jornadas de capacitación, los integrantes del grupo tienen la oportunidad de participar en una o más instancias de la capacitación concretando logros que en alguna medida, no estaban previstos y que alcanzaron a los productores, profesionales y alumnos de los grupos. Se espera que a través de la puesta en marcha del proyecto se obtengan resultados que tiendan a capacitar al productor en el manejo de los rastrojos no sólo del algodonero, sino de todos los residuos de cosecha. Con ello se logrará que el productor comprenda que el manejo de la materia orgánica del suelo, es la herramienta fundamental para mantener e incrementar la fertilidad física y química de su suelo, controlar la erosión, evitar el encostramiento y atenuar el efecto de las labranzas en la formación del piso de arado. Que también el productor observe y comprenda que desmenuzando los residuos de cosecha, contribuye a la eliminación de las principales plagas del algodonero, que pasan el período invernal en los residuos de cosecha que no son desmenuzados y/o en los rebrotes de las plantas.

c.- En definitiva, el principal objetivo del programa, es generar un efecto multiplicador sobre la comunidad rural, que asegure que la práctica que se recomienda, sea incorporada al proceso productivo de conservar el suelo y que mejorará su calidad de vida.

Nota: Este proyecto está subvencionado por la Universidad Nacional del Nordeste a través de la Secretaría General de Extensión Universitaria, en el marco del Programa La Universidad en el Medio.

Publicado en formato digital: Dr. Juan Prause e Ing. Agr. María Josefa Marinich. LA IMPORTANCIA 